\title{
Cáncer y COVID-19, perspectiva.
}

*Correspondencia:

ivanakgm@hotmail.com

Teléfono [593] 042288088

Conflicto de intereses: La autora declaran no tener conflictos de intereses.

Recibido: 10 Agosto 2020 Aceptado: 21 Agosto 2020

Publicado: 30 Agosto 2020

Membrete bibliográfico: García K. Cáncer y Covid-19. Rev. Oncol. Ecu 2019;30(2):92-94.

ISSN: 2661-6653

DOI: https://doi.org/10.33821/475

Copyright García K. Este artículo es distribuido bajo los términos de Creative Commons Attribution License, el cual permite el uso y redistribución citando la fuente y al autor original.

\section{Cancer and COVID-19, perspective.}

\author{
Katherine García Matamoros $1 *$ (id)
}

1. Servicio de Oncología, Solca- Guayaquil.

DOI: $10.33821 / 475$

La ciudad de Guayaquil se convirtió en el epicentro de la pandemia en el Ecuador, el primer caso reportado oficialmente fue en la ciudad de Guayaquil el 29 de febrero del 2020 y el 11 de marzo del 2020 se declara Estado de Emergencia Sanitaria en el Sistema Nacional de Salud [1]. El virus denominado SARS-CoV-2 [2], responsable de la nueva enfermedad COVID19 (Corona Virus Disease 19) [3], llegó como un huracán, nadie se imaginó lo que pasaría en marzo y abril del 2020, la incertidumbre, la cuarentena, el bloqueo, sin estudios clínicos controlados para conocer cuál era la mejor opción de tratamiento, pero el sentido común y las experiencias de otros países que estaban pasando por la tormenta que es el COVID-19, nos ayudaron a tener una perspectiva del problema y a plantearnos objetivos a corto y mediano plazo.

Trabajando en un hospital de tercer nivel, de especialidad en oncología, se preveía el riesgo de desarrollar enfermedad grave con mayor mortalidad, en esta población de pacientes. Ya había sido reportado en China, una serie de 1590 pacientes diagnosticados de COVID-19 con laboratorio y admitidos con enfermedad respiratoria aguda, de estos el 1\% (18 pacientes) tenían diagnóstico de cáncer, cuando se comparó con la población sin cáncer se notó, que los pacientes con cáncer tenían mayor riesgo de desarrollar eventos severos que pacientes sin cáncer y de requerir ventilación mecánica y admisiones en la unidad de cuidados intensivos: $39 \%$ vs $8 \%$ [4]. En una serie de casos reportados de tres centros designados en Wuhan, de 28 pacientes que fueron diagnosticados y hospitalizados por COVID-19 con cáncer, la tasa de mortalidad fue de $28.6 \%$ [5]. Pacientes con cáncer que tuvieron quimioterapia o cirugía dentro de 30 días previos a la infección por el SARS-Cov-2 tienen más riesgo de desarrollar COVID-19 con mayor gravedad. Un estudio multicéntrico de 14 hospitales de Wuhan, enrolo 105 pacientes con cáncer y COVID-19 y señalan que estos experimentaron más infecciones intrahospitalarias, mayor riesgo de muerte, se deterioraron más rápidamente que la población sin cáncer, que los pacientes en estadio metastásico tenían aún más riesgo de muerte, que los canceres hematológicos y el cáncer de pulmón tienen mayor grado de severidad que otros tipos de cáncer, sin embargo la radioterapia no planteo mayor riesgo de fatalidad [6]. En un reporte de casos en Nueva York de pacientes con cáncer y COVID-19 sintomático diagnosticados entre marzo y abril del 2020 , un $40 \%$ fueron hospitalizados, $20 \%$ desarrollaron enfermedad respiratoria severa y $12 \%$ murieron [7]. 
Se han planteado una serie de recomendaciones [8-10], sobre todo en pacientes que se encuentran en tratamiento activo, en el pico más alto de la pandemia, como preferir la quimioterapia oral, neoadyuvancia con inhibidores de aromatasa en cáncer de mama hormonal positivo en pacientes postmenopausicas, mayor uso de estimulantes de colonias de granulocitos, en ciertos tumores vacaciones de quimioterapia, des-intensificar los protocolos de quimioterapia, sin que esto disminuya la efectividad en el grupo de pacientes con intensión curativa, posponer cirugías electivas reconstructivas, en general el enfoque actual es continuar con los tratamientos con el desafío de minimizar la exposición al virus tanto como sea posible [11].

Los sistemas de salud en los países con ingresos económicos bajos y medianos se han visto mucho más abrumados y sobrecargados en la atención de salud durante la pandemia del COVID-19 [12], cada hospital, cada equipo de trabajo ha desarrollado estrategias para minimizar el impacto en los proveedores de salud y en los pacientes. Una cosa queda clara, los gobiernos deben tomar conciencia, que la salud pública debe ser siempre una prioridad.

La telemedicina o salud virtual [13-15] ha innovado la atención, los hospitales al recibir masivamente pacientes se convierten en una fuente de infecciones nosocomiales, para disminuir los contactos innecesarios en casos en los que la visita al hospital es de mayor riesgo, para brindar Servicios y tranquilidad a los pacientes, hemos aprendido a tener nuevos canales de comunicación, gracias a la tecnología e informática, la telemedicina puede ser fortalecida en el futuro y llegar a convertirse en una herramienta muy útil en la vida cotidiana de hospitales y centros de salud.

Las cifras en el futuro nos dirán cuáles fueron los aciertos y los desaciertos, conocemos los retos en la salud pública, como los tiempos de espera para la atención, falta de recursos tanto humanos como de tecnología y de medicamentos, falta de apoyo a la investigación, burocracia que retarda los procesos, es necesario escribir la historia, es necesario conocer las cifras, solo así podemos aprender y mejorar.

\section{Referencias}

1. Ministerio de Salud Pública. Actualización de casos de coronavirus en Ecuador Boletin 2020. [Internet]. Disponible en: https://www.salud.gob.ec/actualizacion-de-casos-de-coronavirus-en-ecuador/

2. Lu R, Zhao X, Li J, Niu P, Yang B, Wu H, et al. Genomic characterisation and epidemiology of 2019 novel coronavirus: implications for virus origins and receptor binding. The Lancet. 22 de febrero de 2020;395(10224):565 74. DOI: $10.1016 /$ S0140-6736(20)30251-8

3. Zhu Na, Zhang D, Wang W, Li X, YangB, Song J, A Novel Coronavirus from Patients with Pneumonia in China, 2019 N Engl J Med 2020; 382:727-733. DOI: 10.1056/NEJMoa2001017

4. Liang W, Guan W, Chen R, Wang W, Li J, Xu K, Li C, Ai Q, Lu W, Liang H, Li S, He J. Cancer patients in SARS-CoV-2 infection: a nationwide analysis in China. Lancet Oncol. 2020 Mar;21(3):335-337. DOI: 10.1016/S14702045(20)30096-6. Epub 2020 Feb 14. PMID: 32066541; PMCID: PMC7159000.

5. Zhang L, Zhu F, Xie L, Wang C, Wang J, Chen R, Jia P, Guan HQ, Peng L, Chen Y, Peng P, Zhang P, Chu Q, Shen Q, Wang Y, Xu SY, Zhao JP, Zhou M. Clinical characteristics of COVID-19-infected cancer patients: a retrospective case study in three hospitals within Wuhan, China. Ann Oncol. 2020 Jul;31(7):894-901. DOI: 10.1016/j.annonc.2020.03.296. Epub 2020 Mar 26. PMID: 32224151; PMCID: PMC7270947. 
6. Dai M, Liu D, Liu M, Zhou F, Li G, Chen Z, et al. Patients with Cancer Appear More Vulnerable to SARS-CoV-2: A Multicenter Study during the COVID-19 Outbreak. Cancer Discov. 2020;10(6):783-791. DOI: 10.1158/2159-8290.CD20-0422. Epub 2020 Apr 28. PMID: 32345594; PMCID: PMC7309152.

7. Robilotti EV, Babady NE, Mead PA, Rolling T, Perez-Johnston R, Bernardes M, et al. Determinants of COVID-19 disease severity in patients with cancer. Nat Med. agosto de 2020;26(8):1218-23. DOI: 10.1038/s41591-020-0979$\underline{0}$

8. Gosain R, Abdou Y, Singh A, Rana N, Puzanov I, Ernstoff MS. COVID-19 and Cancer: a Comprehensive Review. Curr Oncol Rep. 2020 May 8;22(5):53. DOI: 10.1007/s11912-020-00934-7. PMID: 32385672; PMCID: PMC7206576.

9. Al-Quteimat OM, Amer AM. The Impact of the COVID-19 Pandemic on Cancer Patients. Am J Clin Oncol. 2020 Jun;43(6):452-455. DOI: 10.1097/COC.0000000000000712. PMID: 32304435; PMCID: PMC7188063..

10. Marron JM, Joffe S, Jagsi R, Spence RA, Hlubocky FJ. Ethics and Resource Scarcity: ASCO Recommendations for the Oncology Community During the COVID-19 Pandemic. J Clin Oncol 2020;38(19):2201-2205. DOI: $\underline{10.1200 / J C 0.20 .00960}$

11. Jindal V, Sahu KK, Gaikazian S, Siddiqui AD, Jaiyesimi I. Cancer treatment during COVID-19 pandemic. Med Oncol. julio de 2020;37(7):58. DOI: 10.1007/s12032-020-01382-w

12. De Guzman R, Malik M. Dual Challenge of Cancer and COVID-19: Impact on Health Care and Socioeconomic Systems in Asia Pacific. JCO Glob Oncol. 2020 Jun;6:906-912. DOI: 10.1200/G0.20.00227. PMID: $\underline{32589462}$ PMCID: PMC7328111

13. Mann DM, Chen J, Chunara R, Testa PA, Nov O. COVID-19 transforms health care through telemedicine: Evidence from the field. J Am Med Inform Assoc. 2020 Jul 1;27(7):1132-1135. DOI: 10.1093/jamia/ocaa072. PMID 32324855; PMCID: PMC7188161.

14. Hong Z, Li N, Li D, Li J, Li B, Xiong W, Lu L, Li W, Zhou D. Telemedicine During the COVID-19 Pandemic: Experiences From Western China. J Med Internet Res. 2020 May 8;22(5):e19577. DOI: 10.2196/19577. PMID: 32349962; PMCID: PMC7212818.:

15. Contreras CM, Metzger GA, Beane JD, Dedhia PH, Ejaz A, Pawlik TM. Telemedicine: Patient-Provider Clinical Engagement During the COVID-19 Pandemic and Beyond. J Gastrointest Surg. 2020 Jul;24(7):1692-1697. DOI: 10.1007/s11605-020-04623-5. Epub 2020 May 8. PMID: 32385614; PMCID: PMC7206900. 